05

\title{
Теплоэлектродинамические механизмы нарастания вольт-амперных характеристик технических сверхпроводников при крипе магнитного потока
}

\author{
(C) В.P. Романовский \\ Национальный исследовательский центр „Курчатовский институт“, \\ 123182 Москва, Россия \\ e-mail: romanovskii@aol.com
}

(Поступило в Редакцию 28 марта 2016 г.)

Изучены процессы формирования макроскопических состояний сверхпроводящей ленты, индуцируемые при крипе магнитного потока транспортным током. Показано существование характерных значений напряженности электрического поля, зависящих от скорости ввода тока, свойств сверхпроводника, условий охлаждения и свойств стабилизирующей матрицы, которые лежат в основе теплоэлектродинамических механизмов, влияющих на крутизну нарастания вольт-амперных характеристик технических сверхпроводников. Исследованы условия возникновения токовых неустойчивостей с учетом неравномерного распределения температуры по сечению технического сверхпроводника. Сформулированы условия существования вольтамперных характеристик технических сверхпроводников, допускающие стабильный нагрев сверхпроводника до температуры, равной критической. Результаты проведенного исследования необходимо учитывать при измерении вольт-амперных характеристик сверхпроводящих материалов, определении их критических параметров и тока возникновения неустойчивости.

DOI: 10.21883/JTF.2017.01.44018.1823

\section{Введение}

Низкотемпературные и высокотемпературные сверхпроводящие материалы, служащие основой при изготовлении современных технических сверхпроводников (сверхпроводник + матрица или покрытие из несверхпроводящего металла), позволяют создавать электрофизические установки, генерирующие мегавысокие магнитные поля. Поэтому исследованию транспортных свойств технических сверхпроводников уделяется постоянное внимание. Их анализ позволяет выполнить оценку важнейших параметров сверхпроводящих материалов, влияющих на макродинамику электромагнитного поля внутри технических сверхпроводников при крипе магнитного потока $[1,2]$. Поскольку в области высоких магнитных полей магнитная проницаемость сверхпроводников близка к единице $[1,2]$, их главной характеристикой при крипе магнитного потока является вольт-амперная характеристика. В простейшем виде она может быть описана известным степенным уравнением [1]: $E=E_{c}\left(J / J_{c}\right)^{n}$. В этом случае критическая плотность тока сверхпроводника $J_{c}$ вводится как ток, при котором на его вольт-амперной характеристике фиксируется появление априори заданного напряжения $E_{c}$. В то же время при крипе магнитного потока электрическое напряжение, а значит, и тепловые потери неизбежно индуцируются и в докритической области $\left(E<E_{c}, J<J_{c}\right)$. Соответственно определенные таким образом критические свойства сверхпроводника условно описывают его фундаментальные характеристики, поскольку измеряемый критический ток будет зависеть от условий охлаждения, свойств стабилизирующей матрицы, особенностей ввода тока. В связи с этим в настоящей работе обсуждаются теплоэлектродинамические механизмы, лежащие в основе изменения крутизны нарастания вольт-амперной характеристики технических сверхпроводников, которые необходимо учитывать в экспериментах по определению как критических параметров сверхпроводящих материалов, так и тока срыва ввода, после превышения которого возникают неустойчивые состояния.

\section{Постановка задачи}

Исследуем процесс ввода тока в сверхпроводящую ленту $(-a<x<a,-b<y<b, b \gg a)$, помещенную в постоянное внешнее магнитное поле $B$, которое полностью проникло внутрь ленты. Будем полагать, что изменением внешнего магнитного поля и критического тока сверхпроводника в продольном направлении $(-\infty<z<\infty)$ можно пренебречь, и тонкие сверхпроводящие жилы, равномерно распределенные по сечению ленты с коэффициентом заполнения $\eta$, не приводят к возникновению магнитных неустойчивостей. Пусть ток $I$ увеличивается с постоянной скоростью $d I / d t$ от нулевого значения, а на боковых поверхностях сверхпроводника имеет место конвективный теплообмен с коэффициентом теплоотдачи $h$. Предположим, что вольтамперная характеристика сверхпроводника описывается при крипе магнитного потока степенным уравнением, его критическая плотность тока $J_{c}$ линейно уменьшается с ростом температуры $[1,2]$.

Согласно данным допущениям, изменение температуры и напряженности электрического поля в поперечном 
сечении ленты могут быть определены из решения системы одномерных нестационарных уравнений вида [3]

$$
\begin{aligned}
C(T) \frac{\partial T}{\partial t} & =\frac{\partial}{\partial x}\left(\lambda(T) \frac{\partial T}{\partial x}\right)+ \begin{cases}0, & 0<x<x_{p}, \\
E J, & x_{p}<x<a,\end{cases} \\
\mu_{0} \frac{\partial J}{\partial t} & =\frac{\partial^{2} E}{\partial x^{2}}, \quad t>0, \quad 0 \leq x_{p}<x<a
\end{aligned}
$$

при следующих начально-краевых условиях:

$$
\begin{gathered}
T(x, 0)=T_{0}, \quad \frac{\partial T}{\partial x}(0, t)=0, \\
\lambda \frac{\partial T}{\partial x}(a, t)+h\left[T(a, t)-T_{0}\right]=0, \\
E(x, 0)=0, \quad \begin{cases}E\left(x_{p}, t\right)=0, & x_{p}>0 \\
\frac{\partial E}{\partial x}(0, t)=0, & x_{p}=0,\end{cases} \\
\frac{\partial E}{\partial x}(a, t)=\frac{\mu_{0}}{4 b} \frac{d I}{d t} .
\end{gathered}
$$

Здесь $C(T)$ - объемная теплоемкость ленты, $\lambda(T)-$ коэффициент ее теплопроводности, $T_{0}$ - температура хладагента, $a, b$ - геометрические размеры ленты, $x_{p}$ - подвижная координата области проникновения транспортного тока, удовлетворяющая согласно [4] равенству

$$
4 b \int_{x_{p}}^{a} J(x, t) d x=\frac{d I}{d t} t .
$$

В рассматриваемом приближении общая плотность тока $J(x, t)$, введенного в ленту, равна

$$
J=\eta J_{s}+(1-\eta) J_{m},
$$

где плотности токов в сверхпроводящей части ленты $J_{s}$ и в покрытии $J_{m}$ удовлетворяют равенствам

$$
E=E_{c}\left[J_{s} / J_{c}(T, B)\right]^{n}=J_{m} \rho_{m}(T, B) .
$$

Здесь $n-$ показатель нарастания вольт-амперной характеристики сверхпроводника, $E_{c}-$ напряженность электрического поля, используемая для определения критической плотности тока сверхпроводника $J_{c}$. Ее зависимость от температуры и индукции магнитного поля опишем выражением вида $[1,2]$

$$
J_{c}(T, B)=J_{c 0}(B)\left[T_{c B}(B)-T\right] /\left[T_{c B}(B)-T_{0}\right],
$$

в котором $J_{c 0}$ и $T_{c B}-$ известные критические параметры сверхпроводника при заданном внешнем магнитном поле $B$.

Кроме модели (1)-(8) для анализа макроскопических состояний сверхпроводящей ленты могут быть использованы и другие приближения. Режим частичного проникновения тока может быть описан на основе автомодельных решений, предложенных в [4], если при этом температура ленты близка к температуре охладителя. Тогда при $n \geq 10$ распределение напряженности электрического поля и изменение во времени глубины проникновения могут быть вычислены как

$$
\begin{gathered}
E(x, t)= \begin{cases}0, & 0 \leq x \leq x_{p}, \\
\frac{\mu_{0}}{4 b} \frac{d I}{d t}\left(x-x_{p}\right), & x_{p} \leq x \leq a,\end{cases} \\
x_{p}(t)=a-\left(\frac{n+1}{n} t\right)^{\frac{n}{n+1}}\left(\frac{\mu_{0}}{4 b} \frac{d I}{d t}\right)^{\frac{n-1}{n+1}}\left[\frac{E_{c}}{\mu_{0}^{n} \eta^{n} J_{c 0}^{n}}\right]^{\frac{1}{n+1}} .
\end{gathered}
$$

Для упрощенного анализа режима полного проникновения можно воспользоваться стационарной нульмерной моделью, впервые сформулированной в [5] в приближении $d I / d t \rightarrow 0$. В этом случае теплоэлектродинамические состояния сверхпроводящей ленты будут описываться соотношениями [6]

$$
\begin{gathered}
J=\left(\frac{\eta J_{c 0}}{E_{c}^{1 / n}} E^{1 / n}+\frac{1-\eta}{\rho_{m}} E\right) /\left[1+\frac{J_{c 0}}{E_{c}^{1 / n}} \frac{\eta a}{h\left(T_{c B}-T_{0}\right)} E^{(n+1 / n)}\right], \\
T=T_{0}+\frac{E a}{h}\left(\frac{\eta J_{c 0}}{E_{c}^{1 / n}} E^{1 / n}+\frac{1-\eta}{\rho_{m}} E\right) / \\
{\left[1+\frac{J_{c 0}}{E_{c}^{1 / n}} \frac{\eta a}{h\left(T_{c B}-T_{0}\right)} E^{(n+1 / n)}\right] .}
\end{gathered}
$$

Они позволяют найти границу устойчивых токовых состояний при полном проникновении транспортного тока внутрь ленты. А именно напряженность электрического поля $E_{q, 0}$ перед возникновением токовой неустойчивости следует из решения трансцендентного уравнения

$$
\begin{aligned}
\frac{\eta}{n} \frac{J_{c 0}}{E_{c}^{(1 / n)}} & E_{q, 0}^{(1-n) / n}-\left(\eta \frac{J_{c 0}}{E_{c}^{1 / n}}\right)^{2} \frac{a}{h\left(T_{c B}-T_{0}\right)} E_{q, 0}^{2 / n} \\
& =\frac{1-\eta}{\rho_{m}}\left[\frac{\eta}{n} \frac{J_{c 0}}{E_{c}^{1 / n}} \frac{a}{h\left(T_{c B}-T_{0}\right)} E_{q, 0}^{(n+1) / n}-1\right] .
\end{aligned}
$$

При этом соответствующие значения тока срыва $I_{q, 0}$ и температуры сверхпроводника перед возникновением неустойчивости $T_{q, 0}$ равны

$$
\begin{gathered}
I_{q, 0}=4 a b \frac{\eta \frac{J_{c 0}}{E_{c}^{1 / n}} E_{q, 0}^{1 / n}+\frac{1-\eta}{\rho_{m}} E_{q, 0}}{1+\frac{J_{c 0}}{E_{c}^{1 / n}} \frac{\eta a}{h\left(T_{c B}-T_{0}\right)} E_{q, 0}^{(n+1) / n}} \\
T_{q, 0}=T_{0}+\left(T_{c B}-T_{0}\right) \frac{\eta+\frac{1-\eta}{\rho_{m}} \frac{E_{c}^{1 / n}}{J_{c 0}} E_{q, 0}^{(n-1) / n}}{\eta+\frac{h\left(T_{c B}-T_{0}\right)}{a} \frac{E_{c}^{1 / n}}{J_{c 0}} E_{q, 0}^{-(n+1) / n}} .
\end{gathered}
$$

Ниже приведены результаты анализа теплоэлектродинамических процессов, происходящих в ленте $\left(a=0.19 \cdot 10^{-3} \mathrm{~m}, \quad b=2.45 \cdot 10^{-3} \mathrm{~m}\right)$ на основе $\mathrm{Bi}_{2} \mathrm{Sr}_{2} \mathrm{CaCu}_{2} \mathrm{O}_{8}$ с серебряным покрытием при $T_{0}=4.2 \mathrm{~K}, h=10 \mathrm{~W} /\left(\mathrm{m}^{2} \mathrm{~K}\right)$, помещенной в магнитное поле $B=10 \mathrm{~T}$. Исходные параметры ленты, согласно [7], принимались равными: $n=10, E_{c}=10^{-4} \mathrm{~V} / \mathrm{m}$, 


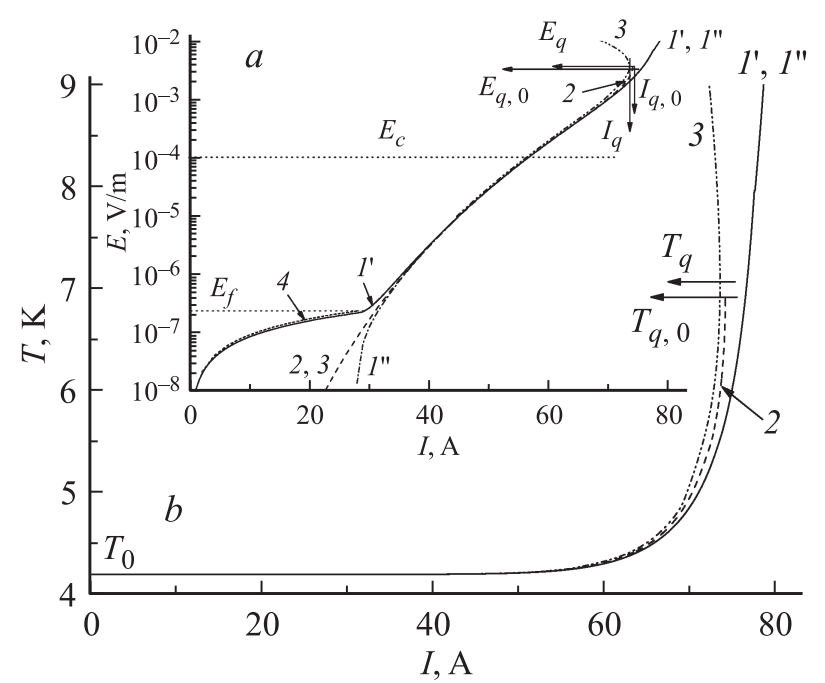

Pис. 1. Статическое и динамическое изменения напряженности электрического поля $(a)$ и температуры $(b)$ сверхпроводящей ленты: $1,1^{\prime}-$ модель (1)-(8), 2 - модель (10), 3 - модель (1)-(8) при $d I / d t \rightarrow 0,4$ - модель (9).

$T_{c B}=26.1 \mathrm{~K}, J_{c 0}=1.52 \cdot 10^{8} \mathrm{~A} / \mathrm{m}^{2}, \eta=0.2$. Температурная зависимость теплоемкости ленты с учетом теплоемкости сверхпроводника $C_{s}$ и матрицы $C_{m}$ определялась как $C(T)=\eta C_{s}(T)+(1-\eta) C_{m}(T)$. При этом температурная зависимость теплоемкости сверхпроводника рассчитывалась по формуле $C_{s}=58.5 T+22 T^{3} \mathrm{~J} /\left(\mathrm{m}^{3} \mathrm{~K}\right)$, $T \leq 10 \mathrm{~K}$. Объемная теплоемкость серебряной матрицы $C_{m}$ была вычислена согласно результатам [8]. Коэффициент теплопроводности ленты вычислялся по формуле $\lambda(T)=2.45 \cdot 10^{-8} T(1-\eta) /(1+\eta) / \rho_{m}(T, B)$, принимая во внимание только удельное сопротивление матрицы $\rho_{m}$ [9]. Зависимость $\rho_{m}$ от температуры и индукции магнитного поля рассчитывалась в соответствии с $[10,11]$ при $\rho_{m}(273 \mathrm{~K}) / \rho_{m}(4.2 \mathrm{~K})=10$ и $\rho_{m}(273 \mathrm{~K})=1.48 \cdot 10^{-8} \Omega \cdot \mathrm{m}$. Скорость ввода тока полагалась равной $d I / d t=10 \mathrm{~A} / \mathrm{s}$.

\section{Результаты}

Обсудим основные физические закономерности формирования стабильных и нестабильных ветвей вольтамперных характеристик сверхпроводящей ленты, учитывая с помощью предложенных выше моделей особенности изменения ее температурного и электрического полей, индуцированных транспортным током.

На рис. 1 изображено изменение напряженности электрического поля и температуры, которое будет иметь место на поверхности и в центре сверхпроводящей ленты - кривые $l^{\prime}$ и $l^{\prime \prime}$ соответственно. Здесь же для иллюстрации пространственных особенностей формирования теплоэлектродинамических состояний ленты, которые имеют место как при устойчивых, так и неустойчивых состояниях, приведены вольт-амперная и температурно-амперная характеристики ленты, рассчитанные согласно (10) (кривые 2), т.е. в предположении равномерного распределения температуры и электрического поля по сечению ленты. Соответственно на рис. 1 изображены напряженность электрического поля $E_{q, 0}$, ток $I_{q, 0}$ и температура $T_{q, 0}$, определяющие границу устойчивости пространственно однородных стационарных состояний ленты, которые следуют из (11)-(13). Кроме этого, кривые 3 соответствуют статическим $E(I)$ и $T(I)$ характеристикам ленты, определенным в предположении бесконечно медленного ввода тока $(d I / d t \rightarrow 0)$. В этом случае теплоэлектродинамические состояния ленты определялись на основе решения задачи (1)-(8) при $t \rightarrow \infty$, т. е. решалась соответствующая краевая задача. Кривая 4 соответствует состояниям, расчет которых был выполнен на основе автомодельного приближения (9). Рис. 1 наглядно демонстрирует характерные физические особенности нарастания температуры и напряженности электрического поля внутри технических сверхпроводников.

На стадии неполного проникновения тока по вполне понятным причинам внутри ленты наблюдается неравномерное распределение электрического поля по ее сечению. При этом данный режим сопровождается не только практически однородным распределением температуры, но и характеризуется ее слабым отклонением от температуры хладагента (рис. $1, b$ ). Это объясняется тем, что основная часть введенного тока течет по сверхпроводящей части ленты, и поэтому тепловыделения незначительны. Тогда, согласно (9), нетрудно найти значение напряженности электрического поля $E_{f}$ на поверхности ленты в момент времени, когда ток полностью проникнет в ленту. Оно равно

$$
E_{f}=\frac{\mu_{0}}{4 b} \frac{d I}{d t} a .
$$

Данная формула показывает как скорость ввода тока и геометрия ленты влияют на формирование электродинамических состояний ленты в режиме частичного проникновения, что необходимо учитывать при измерении критического тока сверхпроводника. А именно значение $E_{f}$ будет увеличиваться с увеличением толщины ленты, но будет тем меньше, чем больше ее ширина. Как следствие, неравномерное распределение электрического поля по сечению ленты будет наблюдаться не только на начальной стадии режима полного проникновения тока внутрь ленты, но и может иметь место в области напряжений, обычно используемых при измерении критического тока. Данная тенденция будет тем заметнее, чем больше скорость ввода тока. Таким образом, значение $E_{f}$, во-первых, позволяет оценить границу электрических напряжений, ниже которой измерение критического тока сверхпроводника некорректно, вовторых, показывает, что после его превышения начальная стадия полного проникновения тока характеризуется неравномерно распределенным электрическим полем. Как следует из рис. $1, a$, распределение электрического 
поля в режиме полного проникновения тока, близкое к равномерному, будет наблюдаться тогда, когда соблюдается простейшее условие $E_{f} \ll E_{c}$, т. е., например, при $d I / d t \ll 4 b E_{c} / \mu_{0} a$. В то же время видно, что это условие не является строгим. Распределение электрического поля по сечению ленты будет равномерным только в предельном случае $d I / d t \rightarrow 0(t \rightarrow \infty)$. Этот вывод следует из уравнения (2) и граничных условий (4).

В области закритических электрических полей $(E>$ $\left.>E_{c}\right)$ нестационарные $E(I)$ - и $T(I)$-характеристики ленты (кривые $1^{\prime}, 1^{\prime \prime}$ ) количественно и качественно отличаются от зависимостей, рассчитанных в стационарном приближении (кривые 2 и 3). Эта особенность связана с конечным стабильным перегревом ленты и влиянием ее теплоемкости на протекающие в ней нестационарные электродинамические процессы. Согласно [3], нарастание зависимостей $E(I)$ и $T(I)$ при непрерывном вводе тока будет зависеть от интенсивности увеличения температуры сверхпроводника даже на стадии стабильных состояний. Поэтому наклон кривых $1^{\prime}, 1^{\prime \prime}$ всегда положителен и будет уменьшаться с увеличением скорости ввода тока в силу соответствующего увеличения с температурой теплоемкости ленты. Именно поэтому нестационарные вольт-амперные характеристики сверхпроводников не позволяют определить значение тока возникновения неустойчивости. Этот вывод объясняет, почему при экспериментальном измерении тока срыва используется импульсный метод ввода тока с последующим измерением изменяющегося во времени индуцированного электрического напряжения. Но и при этом условие

$$
\partial E / \partial J \rightarrow \infty,
$$

позволяющее в приближении равномерного распределения тока и температуры найти граничные значения $E_{q, 0}, I_{q, 0}$ и $T_{q, 0}$, не соблюдается, как это следует из pис. 1, $a$. Граница возникновения токовой неустойчивости в общем случае, учитывающем неоднородность макроскопического состояния ленты, в стационарном приближении $(t \rightarrow \infty)$ следует из условия

$$
\partial E / \partial I \rightarrow \infty \text {. }
$$

Отличие условия (16) от условия (15) показывает, что в экспериментах теплоэлектродинамические состояния сверхпроводящих сред зависят от характера распределения тока и температуры по всему сечению ленты. Из сказанного следует, что расчетный диапазон стабильных значений токов, определенный с учетом неравномерного распределения температуры по сечению ленты, даже когда оно незначительно, будет меньше по сравнению с аналогичными расчетными значениями, полученными в рамках пространственно однородной модели. Соответствующие параметры срыва, рассчитанные в рамках нуль-мерной и одномерной моделей, изображены на рис. 1.

На рис. 2 построены устойчивые и неустойчивые ветви вольт-амперной и температурно-амперной харак-

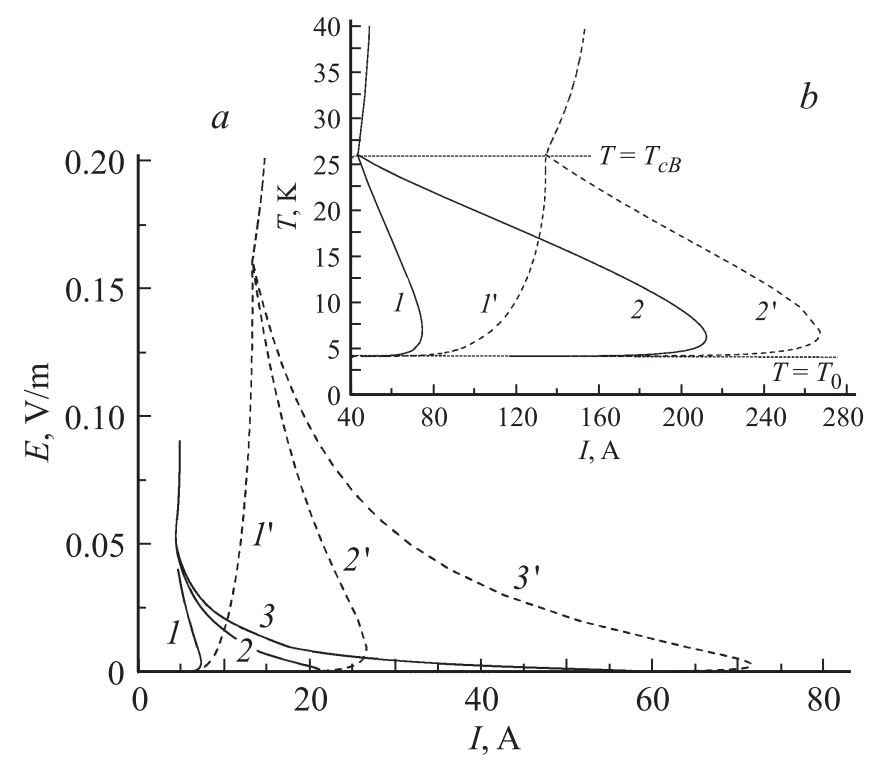

Рис. 2. Влияние коэффициента теплоотдачи и критического тока на вольт-амперную $(a)$ и температурно-амперную $(b)$ характеристики: $--h=10 \mathrm{~W} / \mathrm{m} \cdot \mathrm{K},-\cdots-h=100 \mathrm{~W} / \mathrm{m} \cdot \mathrm{K}$; $1,1^{\prime}-J_{c 0}=1.5 \cdot 10^{8} \mathrm{~A} / \mathrm{m}^{2}, 2,2^{\prime}-J_{c 0}=5 \cdot 10^{8} \mathrm{~A} / \mathrm{m}^{2}, 3,3^{\prime}-$ $J_{c 0}=15 \cdot 10^{8} \mathrm{~A} / \mathrm{m}^{2}$.

теристик, рассчитанные для рассматриваемой ленты в стационарном приближении при варьировании коэффициента теплоотдачи и критического тока сверхпроводника. Представленные результаты, полученные на основе использования одномерного стационарного приближения, т.е. при $d I / d t \rightarrow 0$, показывают, что при определенных условиях стационарные вольт-амперная и температурно-амперная характеристики технических сверхпроводников могут нарастать без катастрофического развития неустойчивости (кривая $1^{\prime}$ ). Соответственно стационарные $E(I)$ - и $T(I)$-характеристики будут иметь положительный наклон во всем диапазоне изменения транспортного тока даже при нагреве сверхпроводника до температуры, равной критической. Как показывают расчеты, подобные состояния будут также наблюдаться и при варьировании количества стабилизирующего покрытия. Данные выводы наглядно демонстрируют, насколько важно для анализа токовых состояний технических сверхпроводников, прежде всего изготовленных из высокотемпературных сверхпроводящих материалов, знать их температуру, которая может устойчиво повышаться вплоть до критической.

В целом рис. 1,2 строго доказывают существование стабильных закритических режимов, когда у технических сверхпроводников токовые неустойчивости при крипе магнитного потока могут происходить при значениях напряженности электрического поля и токах, значительно превышающих условно заданные величины $E_{c}$ и $J_{c}$. Именно поэтому имеет место заметный стабильный перегрев сверхпроводника. Однако последний приводит 
к тепловой деградации токонесущей способности технических сверхпроводников. Действительно, как следует из рис. 2 , при $h=10 \mathrm{~W} /\left(\mathrm{m}^{2} \mathrm{~K}\right)$ увеличение критической плотности тока в 3.33 раза (переход от состояния 1 к состоянию 2) приводит к увеличению тока срыва в 2.85 раза, увеличение критической плотности тока в 3 раза (переход от состояния 2 к состоянию 3) приводит к увеличению тока срыва в 2.73 раза, а при увеличении критической плотности тока в 10 раз (переход от состояния 1 к состоянию 3) - только в 7.81 раз. Кроме этого, деградация также усиливается с улучшением условий теплоотдачи: переход от состояния $2^{\prime}$ к состоянию $3^{\prime}$, когда при $h=100 \mathrm{~W} /\left(\mathrm{m}^{2} \mathrm{~K}\right)$ критическая плотность тока увеличивается в 3 раза, сопровождается увеличением тока срыва в 2.69 раза, то есть меньше, чем при соответствующем переходе от состояния 2 к состоянию 3. Таким образом, увеличение тока срыва всегда меньше соответствующего увеличения критического тока сверхпроводника. Причем отличие тем заметнее, чем выше критическая плотность тока сверхпроводника и коэффициент теплоотдачи.

Для того, чтобы описать обсужденные особенности формирования вольт-амперной и температурноамперной характеристик технических сверхпроводников, которые будут наблюдаться в режиме полного проникновения тока, выполним их анализ в безразмерном стационарном приближении. В этом случае, как следует из уравнения (2) и граничных условий (4), распределение напряженности электрического поля по сечению ленты становится равномерным. Введем безразмерные переменные, а именно безразмерную температуру $\theta=\left(T_{c B}-T\right) /\left(T_{c B}-T_{0}\right)$, безразмерную пространственную координату $X=x / a$, безразмерное термическое сопротивление ленты $H=h a / \lambda$, предполагая коэффициент теплопроводности ленты постоянным. Соответственно необходимо решить следующую краевую задачу:

$$
\begin{aligned}
& \frac{d^{2} \theta}{d X^{2}}-\theta \frac{E_{c}}{E_{\lambda}}\left(\frac{E}{E_{c}}\right)^{(n+1) / n}-\frac{E^{2}}{E_{\lambda} E_{\eta}}=0, \\
& \frac{d \theta}{d X}(0)=0, \quad \frac{d \theta}{d X}(1)+H \theta(1)=H .
\end{aligned}
$$

Здесь

$$
E_{\eta}=\frac{\eta J_{c 0} \rho_{m}}{1-\eta}, \quad E_{\lambda}=\frac{\lambda\left(T_{c B}-T_{0}\right)}{\eta J_{c 0} a^{2}}, \quad E_{h}=\frac{h\left(T_{c B}-T_{0}\right)}{\eta J_{c 0} a} .
$$

Причем имеют место равенства: $H=E_{h} / E_{\lambda}, \alpha=E_{\eta} / E_{h}$, где $\alpha$ - параметр Стекли [1,2].

Из (17)-(18) следует, что у технических сверхпроводников существуют характерные значения напряженности электрического поля $\left(E_{\eta}, E_{\lambda}\right.$ и $\left.E_{h}\right)$, влияющие на интенсивность нарастания их температуры, а значит, на крутизну нарастания вольт-амперной характеристики. Их значения зависят от условий отвода тепла в хладагент, тепловых и электрических свойств матрицы, критических параметров сверхпроводника. Соответственно будут иметь место различные механизмы формирования теплоэлектродинамических состояний технических сверхпроводников. Обсудим их на качественном уровне строгости.

При $E_{\eta} \rightarrow \infty$ механизм деления тока между сверхпроводником и покрытием не будет влиять на $E(I)$ и $T(I)$-характеристики. Значит $E_{\eta}-$ характерное значение напряженности электрического поля, определяющее границу электрических напряжений, после превышения которой будет иметь место влияние стабилизирующего покрытия на развитие тепловых и электродинамических процессов. Определяющим параметром, влияющим на значения $E_{\eta}$, является удельное электрическое сопротивление покрытия.

При $E_{\lambda} \rightarrow \infty$ распределение температуры по сечению ленты будет равномерным. Поэтому $E_{\lambda}-$ характерная напряженность электрического поля, которая влияет на пространственный характер распределения температуры по сечению ленты, зависящий от количества теплоты, отводимой теплопроводностью ленты. В этом случае параметром, определяющим изменение $E_{\lambda}$, является коэффициент ее теплопроводности.

При $E_{h} \rightarrow \infty$ вольт-амперная характеристика ленты носит изотермический характер $\left(T=T_{0}\right)$. Следовательно, $E_{h}$ - характерная напряженность электрического поля, позволяющая оценить влияние интенсивности внешнего охлаждения ленты (коэффициента теплоотдачи) на характер нарастания ее вольт-амперной характеристики и учесть отклонение электродинамических состояний ленты от состояний, которые существовали бы при идеальных условиях охлаждения.

Варьирование остальных параметров (критической плотности тока сверхпроводника и его критической температуры, коэффициента заполнения ленты сверхпроводником, температуры хладагента, толщины ленты) будет сопровождаться взаимосвязанным изменением значений $E_{\eta}, E_{\lambda}$ и $E_{h}$. Соответственно будет иметь место усиление или уменьшение роли того или иного механизма формирования вольт-амперной характеристики технического сверхпроводника. Например, при увеличении критической плотности тока сверхпроводника увеличивается $E_{\eta}$, но уменьшаются $E_{\lambda}$ и $E_{h}$. В результате, теплоэлектродинамические состояния технических сверхпроводников будут меньше зависеть от удельного электрического сопротивления покрытия. Но они будут характеризоваться более неоднородным распределением температуры, что будет оказывать более существенное влияние на происходящие электродинамические процессы. Увеличение коэффициента заполнения также будет приводить к увеличению влияния температуры и характера ее распределения на вид вольт-амперной характеристики.

Особо отметим вклад тепловых механизмов (кондуктивного и конвективного) в нарастание вольт-амперной характеристики. Их роль будет зависеть от различия между значениями $E_{\lambda}$ и $E_{h}$. Действительно, если $E_{h} \ll E_{\lambda}$, то пространственно-однородное нараста- 


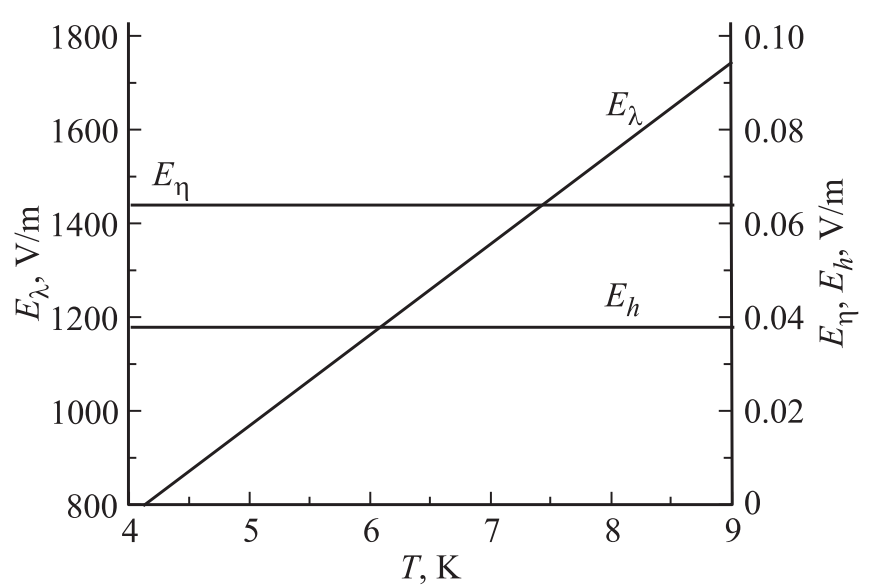

Рис. 3. Влияние температуры на характерные значения напряженности электрического поля.

ние вольт-амперной характеристики технического сверхпроводника в диапазоне электрических полей $E \ll E_{h}$ будет происходить практически изотермически. Соответственно при $E_{h} \ll E \ll E_{\lambda}$ изменение ее теплоэлектродинамических состояний будет носить неизотермический характер. Но при $E \gg E_{\lambda}$ на стационарную вольт-амперную характеристику ленты будет оказывать влияние неравномерное распределение температуры по его сечению. В то же время при $E_{\lambda} \ll E_{h}$ электродинамические процессы, происходящие в ленте, также будут зависеть от пространственной неоднородности температурного поля. Поэтому, в частности, при $E_{\lambda} \ll E_{h} \ll E$ анализ электродинамических состояний сверхпроводника необходимо выполнять с учетом неравномерного распределения температуры по его сечению, т.е. принимать во внимание тепловой размерный эффект.

Зависимости $E_{\eta}, E_{\lambda}$ и $E_{h}$ от температуры для рассматриваемой ленты изображены на рис. 3. Видно, что имеет место следующее соотношение: $E_{q}<E_{h}<E_{\eta} \ll E_{\lambda}$. Оно объясняет, почему распределение температуры по сечению рассматриваемой ленты практически однородное, деление тока не оказывает реального влияния на стабильное формирование теплоэлектродинамических состояний ленты, а срыв ввода тока происходит при закритических параметрах.

Решением краевой задачи (17) является функция

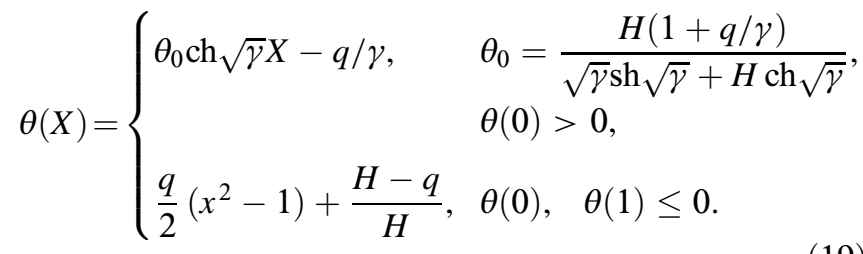

В нуль-мерном приближении (10) однородное распределение температуры по сечению ленты относительно используемых безразмерных переменных описывается следующими выражениями:

$$
\theta= \begin{cases}(H-q) /(H+\gamma), & \theta>0, \\ (H-q) / H, & \theta \leq 0\end{cases}
$$

Здесь

$$
\gamma=\frac{E_{c}}{E_{\lambda}}\left(\frac{E}{E_{c}}\right)^{(n+1) / n}, \quad q=\frac{E^{2}}{E_{\eta} E_{\lambda}} .
$$

При этом имеет место связь $q=\varepsilon \gamma^{2 n /(n+1)}$, где $\varepsilon=$ $=E_{c} / E_{\eta}\left(E_{\lambda} / E_{c}\right)^{(n-1) /(n+1)}$.

По своему физическому смыслу параметры $\gamma$ и $q$ описывают влияние кондуктивного механизма переноса тепла и механизма деления тока на протекающие в ленте теплоэлектродинамические процессы соответственно. В частности, согласно (19), нетрудно найти, что при $\gamma \ll 1$, т.е. при эффективном переносе тепла за счет теплопроводности ленты $\left(E \ll E_{\lambda}\right)$, внутри нее будут иметь место состояния с равномерным распределением температуры. Соответственно выражения (19) переходят в (20). При

$$
\frac{q}{\gamma}=\frac{E_{c}}{E_{\eta}}\left(\frac{E}{E_{c}}\right)^{(n-1) / n} \ll 1,
$$

т.е. при $E \ll E_{\eta}$, основная часть тока будет протекать в сверхпроводнике, и влияние покрытия будет незначительным.

Формула (19) описывает только два тепловых режима, которые будут иметь место при вводе тока в технический сверхпроводник. Прежде всего это режим, когда при $\theta(0)>0$ сверхпроводимость сохраняется во всем объеме ленты. Если $\theta(0)<0$, то центральная часть ленты начинает переходить в нормальное состояние, и при $\theta(1)<0$ сверхпроводящее состояние ленты полностью разрушается. Математическое описание режимов, когда внутри ленты могут стабильно существовать сверхпроводящее и нормальное состояния, весьма громоздко, и оно не приведено. В то же время, как показывают расчеты, для практически важных режимов, а именно при $H \ll 1$, различие в условиях, учитывающих существование и $\theta(0)$, и $\theta(1)$, незначительно. Поэтому формула (19) описывает состояния, когда предполагается, что при $\theta(0)<0$ лента полностью переходит в нормальное состояние.

Из (19) и (20) нетрудно найти критериальные соотношения, при которых будет выполняться условие $\theta=0$, описывающее границу состояний, когда сверхпроводящие свойства ленты сохраняются во всем ее объеме. Относительно введенных безразмерных параметров оно записывается в виде

$$
H=\varepsilon \gamma^{\frac{3 n-1}{2(n+1)}} \operatorname{sh} \sqrt{\gamma} /\left[1+\varepsilon \gamma^{\frac{n-1}{n+1}}(1-\operatorname{ch} \sqrt{\gamma})\right]
$$

в рамках одномерного приближения и

$$
H=\varepsilon \gamma^{\frac{2 n}{n+1}}
$$

в рамках нуль-мерного приближения. 


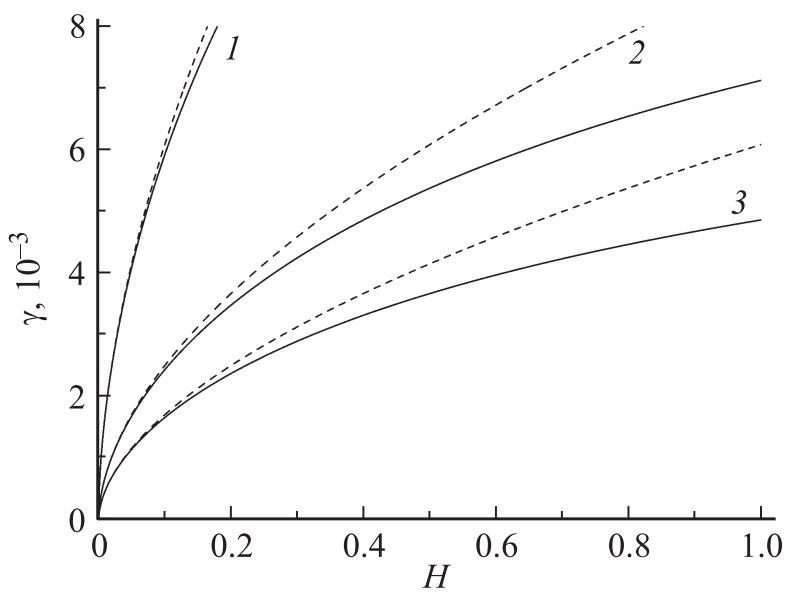

Рис. 4. Зависимость предельно допустимых значений параметpa $\gamma$ от термического сопротивления ленты, после превышения которого весь ток будет стабильно течь только в покрытии: $1-E_{\eta}=0.05 \mathrm{~V} / \mathrm{m}, 2-E_{\eta}=0.01 \mathrm{~V} / \mathrm{m}, 3-E_{\eta}=0.005 \mathrm{~V} / \mathrm{m}$, - - одномерное приближение, - - - — нуль-мерное приближение.

Выражения (21) и (22) позволяют оценить напряженность электрического поля и ток, после превышения которых сверхпроводящие свойства технических сверхпроводников будут разрушены без их скачкообразного перехода в нормальное состояние. Соответствующие значения $\gamma$ как функции термического сопротивления приведены на рис. 4. Расчеты были выполнены при $E_{\lambda}=10^{3} \mathrm{~V} / \mathrm{m}$ и варьировании $E_{\eta}$. Видно, что $H \ll 0.1$, значения $\gamma$ не только много меньше единицы, но и с удовлетворительной точностью могут быть описаны нуль-мерной моделью. С другой стороны, из рис. 4 следует, что неоднородность распределения температуры по сечению технических сверхпроводников необходимо учитывать при $H \gg 0.1$. Соответственно эффект неоднородного распределения температуры будет оказывать существенное влияние на формирование теплоэлектродинамических состояний интенсивно охлаждаемых массивных токонесущих элементов.

В размерном виде стационарное неоднородное распределение температуры по сечению ленты при полном проникновении тока описывается выражением

$$
\begin{aligned}
& T(x)=T_{c B} \\
& -\left(T_{c B}-T_{0}\right) \begin{cases}\theta_{0} \operatorname{ch} \sqrt{\gamma} X-q / \gamma, & T(0)<T_{c B}, \\
\frac{q}{2}\left(X^{2}-1\right)+\frac{H-q}{H}, & T(0) \geq T_{c B} .\end{cases}
\end{aligned}
$$

В этом случае связь между вводимым током на единицу ширины ленты и напряженностью электрического поля, определяемая с учетом симметрии рассматриваемой задачи как

$$
I^{*}(E)=2 \int_{0}^{\alpha} J(E) d x
$$

записывается в виде

$$
I^{*}= \begin{cases}2 \eta J_{c 0} a\left(\frac{E}{E_{c}}\right)^{1 / n} \frac{1+q / \gamma}{\sqrt{\gamma} / \mathrm{th} \sqrt{\gamma}+\gamma / H}, & T(0)<T_{c B}, \\ 2 \eta J_{c 0} a \frac{E}{E_{\eta}}, & T(0) \geq T_{c B} .\end{cases}
$$

Соответствующие выражения, которые следуют из нульмерного приближения, имеют вид

$$
\begin{gathered}
T= \begin{cases}T_{c B}-\left(T_{c B}-T_{0}\right) \frac{H-q}{H+\gamma}, & T<T_{c B}, \\
T_{c B}-\left(T_{c B}-T_{0}\right)\left(1-\frac{q}{H}\right), & T \geq T_{c B},\end{cases} \\
I^{*}= \begin{cases}2 \eta J_{c 0} a\left(\frac{E_{\lambda}}{E_{c}}\right)^{1 /(n+1)} \frac{1+q / \gamma}{1+\gamma / H}, & T<T_{c B}, \\
2 \eta J_{c 0} a \frac{E}{E_{\eta}}, & T \geq T_{c B} .\end{cases}
\end{gathered}
$$

Для состояний, удовлетворяющих условию $\gamma \ll 1$, выражения (25) и (26) позволяют выписать более строгие оценки диапазона значений напряженности электрических полей, когда процесс ввода тока имеет изотермический характер или роль покрытия пренебрежимо мала. Опуская очевидные преобразования, сформулируем их.

Вольт-амперная характеристика технического сверхпроводника будет близка к изотермической при выполнении условия

$$
\frac{T_{c B}-2 T_{0}}{T_{0}} \frac{E}{E_{h}}\left(\frac{E}{E_{c}}\right)^{1 / n}+\frac{T_{c B}-T_{0}}{T_{0}} \frac{E^{2}}{E_{h} E_{\eta}} \ll 1 .
$$

При $T_{c B}>2 T_{0}$ оно может быть записано в более упрощенном виде, а именно

$$
\frac{E^{2}}{E_{h} E_{\eta}} \ll 1
$$

Матрица не будет оказывать влияние на крутизну нарастания вольт-амперной характеристики, если

$$
\frac{E}{E_{\eta}}\left(\frac{E_{c}}{E}\right)^{1 / n} \ll 1 .
$$

(Данная оценка уже была получена выше в рамках одномерного приближения.) В этом случае температура сверхпроводника будет незначительно отличаться от температуры хладагента, когда будет соблюдаться дополнительное условие

$$
\frac{T_{c B}-2 T_{0}}{T_{0}} \frac{E}{E_{h}}\left(\frac{E}{E_{c}}\right)^{1 / n} \ll 1 .
$$

При $T_{c B}>3 T_{0}$ оно преобразуется к виду

$$
\frac{E}{E_{h}}\left(\frac{E}{E_{c}}\right)^{1 / n} \ll 1
$$


Записанные оценки приводят к практически важному выводу: чем меньше разница между критической температурой сверхпроводника и температурой хладагента, тем больше влияние покрытия и выше стабильный разогрев сверхпроводника. Он имеет особое значение для высокотемпературных сверхпроводников, когда их режимы характеризуются высокими рабочими температурами.

На рис. 5,6 построены зависимости $\gamma$ и $\Theta=1-\theta$ от безразмерного параметра $\iota=I^{*} /\left(2 a \eta J_{c 0}\right)$, т. е. обоб-

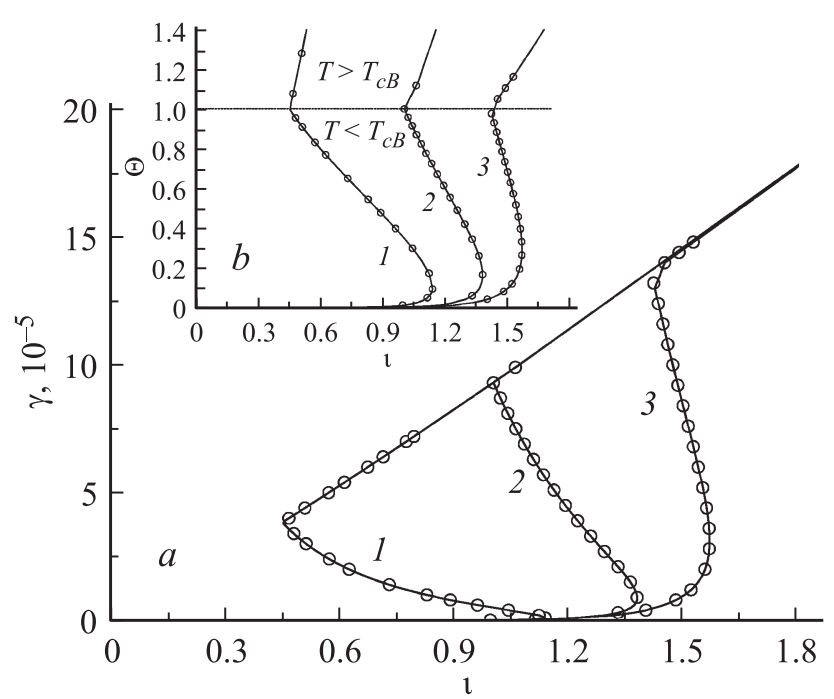

Рис. 5. Безразмерные вольт-амперная и температурноамперная характеристики сверхпроводящей ленты при варьировании $E_{h}: 1-E_{h}=0.01 \mathrm{~V} / \mathrm{m}, \alpha=5 ; 2-E_{h}=0.05 \mathrm{~V} / \mathrm{m}$, $\alpha=1 ; 3-E_{h}=0.1 \mathrm{~V} / \mathrm{m}, \alpha=0.5 ;-$ одномерное приближение, о - нуль-мерное приближение.

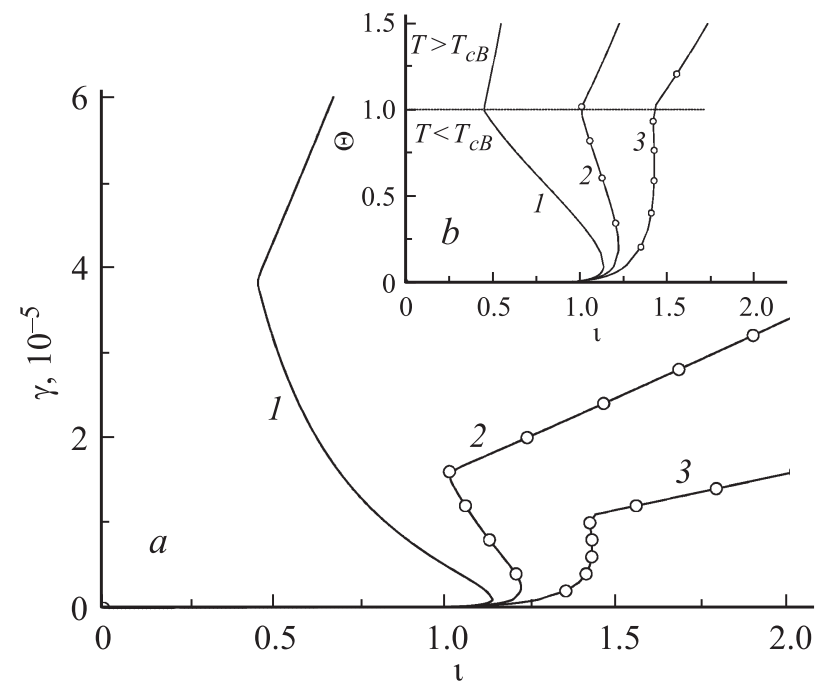

Рис. 6. Безразмерные вольт-амперная и температурноамперная характеристики сверхпроводящей ленты при варьировании $E_{\eta}: 1-E_{\eta}=0.05 \mathrm{~V} / \mathrm{m}, \alpha=5 ; 2-E_{\eta}=0.01 \mathrm{~V} / \mathrm{m}$, $\alpha=1 ; 3-E_{\eta}=0.005 \mathrm{~V} / \mathrm{m}, \alpha=0.5 ;-$ одномерное приближение, о - нуль-мерное приближение.

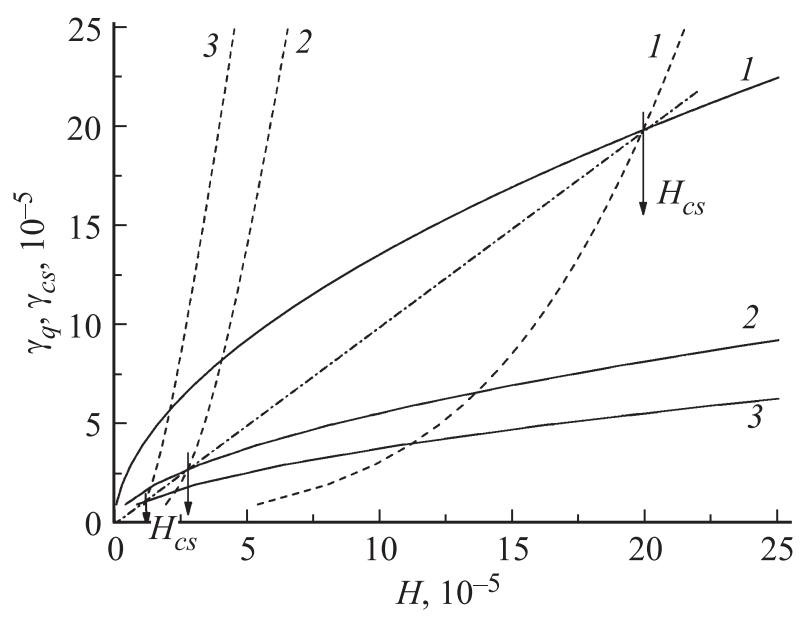

Рис. 7. Влияние безразмерного термического сопротивления ленты на значения $\gamma_{q}(--)$ и $\gamma_{h}(-): 1-E_{\eta}=0.05 \mathrm{~V} / \mathrm{m}, \alpha=5$; $2-E_{\eta}=0.01 \mathrm{~V} / \mathrm{m}, \alpha=1 ; 3-E_{\eta}=0.005 \mathrm{~V} / \mathrm{m}, \alpha=0.5$.

щенные стационарные вольт-амперные и температурноамперные характеристики ленты, рассчитанные в предположении неравномерного и равномерного распределений температуры по ее сечению. Выполненные расчеты основывались на использовании формул $(21)-(26)$ соответственно. При этом, исходя из результатов, приведенных на рис. 3 , полагалось, что $E_{\lambda}=10^{3} \mathrm{~V} / \mathrm{m}$, а значения $E_{h}$ и $E_{\eta}$ варьировались. А именно кривые, изображенные на рис. 5 , были получены при $E_{\eta}=0.05 \mathrm{~V} / \mathrm{m}$ и изменении $E_{h}$, а на рис. $6-$ при $E_{h}=0.01 \mathrm{~V} / \mathrm{m}$ и варьировании $E_{\eta}$.

Представленные результаты, во-первых, строго доказывают вышесделанные выводы, когда для изначально заданных исходных параметров учет в расчетах изменения теплового состояния ленты в ее поперечном сечении практически не приводит к отличию от соответствующих расчетов, выполненных в рамках нуль-мерной модели. Имеет силу более общий вывод, нуль-мерная модель позволяет адекватно описать теплоэлектродинамические состояния, наблюдаемые в экспериментах, если $\gamma \ll 1$ и $H \ll 1$. Во-вторых, токовые режимы могут быть стабильны в закритической области $(l>1)$. Эта закономерность тем заметнее, чем выше $E_{h}$ или ниже $E_{\eta}$. В-третьих, как уже отмечалось, у технических сверхпроводников могут существовать стабильные стационарные вольтамперные и температурно-амперные характеристики с положительным наклоном во всем диапазоне изменения их температуры вплоть до критической температуры сверхпроводника. Другими словами, в этих случаях переход из сверхпроводящего состояния в нормальное может происходить без скачкообразного возникновения токовых неустойчивостей только после достижения критической температуры сверхпроводника. Данные выводы значительно расширяют диапазон практического использования современных технических сверхпроводников.

Согласно условию (16), нетрудно найти искомые параметры срыва: $\gamma_{q}$ - напряженность электрического 
поля перед срывом и $\iota_{q}-$ ток срыва. В терминах используемых безразмерных переменных значение $\gamma_{q}$ в рамках одномерной модели определяется из решения уравнения

$$
H=\frac{\frac{2 n}{n+1} \sqrt{\gamma_{q}} \operatorname{th} \sqrt{\gamma_{q}}\left(1+\frac{\varepsilon}{n} \gamma^{\frac{n-1}{n+1}}\right)}{\frac{2 \sqrt{\gamma_{q}}}{\operatorname{ch} 2 \sqrt{\gamma_{q}}}-\frac{n-1}{n+1}+\varepsilon \gamma^{\frac{n-1}{n+1}}\left(\frac{2 \sqrt{\gamma_{q}}}{\operatorname{sh} 2 \sqrt{\gamma_{q}}}+\frac{n-1}{n+1}\right)} .
$$

В нуль-мерном приближении значение $\gamma_{q, 0}$ удовлетворяет равенству

$$
H=\gamma_{q, 0} \frac{n+q / \gamma_{q, 0}}{1+n q / \gamma_{q, 0}} .
$$

Соответственно токи срыва, определенные согласно одномерному и нуль-мерному приближениям, равны

$$
\begin{gathered}
\iota_{q}=\left(\frac{E_{\lambda}}{E_{c}} \gamma_{q}\right)^{1 /(n+1)} \frac{1+q / \gamma_{q}}{\sqrt{\gamma_{q}} / \operatorname{th} \sqrt{\gamma_{q}}+\gamma_{q} / H}, \\
\iota_{q, 0}=\left(\frac{E_{\lambda}}{E_{c}} \gamma_{q, 0}\right)^{1 /(n+1)} \frac{1+q / \gamma_{q, 0}}{1+\gamma_{q, 0} / H} .
\end{gathered}
$$

Зависимость $\gamma_{q}$ от термического сопротивления $H$ для различных значений $E_{\eta}$ показана на рис. 7. Расчеты проводились при $E_{\lambda}=10^{3} \mathrm{~V} / \mathrm{m}$ и $E_{h}=0.05 \mathrm{~V} / \mathrm{m}$ для пространственно-однородных теплоэлектродинамических состояний. Здесь же изображены кривые $\gamma_{n}(H)$, рассчитанные согласно (22) и тем самым соответствующие тепловым состояниям ленты, когда ее температура становится равной критической. Пересечение кривых $\gamma_{q}(H)$ и $\gamma_{n}(H)$ позволяет найти такие значения $H$ и $\gamma$, после превышения которых технические сверхпроводники будут находиться в нормальном состоянии без возникновения токовой неустойчивости. Согласно нульмерному приближению, состояния $\gamma_{q}\left(H_{c s}\right)=\gamma_{c s}\left(H_{c s}\right)$ приводят к условию

$$
\gamma=q=H_{c s}
$$

и на рис. 7 изображены штрихпунктирной прямой. Относительно размерных переменных оно записывается в виде

Поэтому при

$$
E_{c}^{2}=E_{\eta}^{n+1} / E_{h}^{n-1}
$$

$$
E_{c}^{2}>E_{\eta}^{n+1} / E_{h}^{n-1}
$$

вводимые токи будут стабильно течь только в покрытии. С точки зрения теории тепловой стабилизации [1], это условие аналогично так называемому условию полной стабильности композитного сверхпроводника. Как известно, оно выполняется для всех значений $\alpha$, равных или меньших 1. Но учет крипа приводит к иным значениям параметра Стекли, при которых будет иметь место условие полной стабильности технических сверхпроводников. Запишем его в виде

$$
\alpha<\left(\frac{E_{c}^{2}}{E_{\eta} E_{h}}\right)^{1 / n}=\left[\frac{a(1-\eta) E_{c}^{2}}{\rho_{m} h\left(T_{c B}-T_{0}\right)}\right]^{1 / n} .
$$

Из данного условия следует, что при крипе магнитного потока полная стабильность будет соблюдаться при значениях $\alpha$, которые должны быть заведомо меньше 1 , если $E_{c}<\left(E_{\eta} E_{h}\right)^{1 / 2}$, что выполняется для рассмотренных выше состояний. Действительно, как следует из рис. 5,6, полная стабильность не наблюдается даже для режимов, у которых $\alpha=0.5$. В то же время при $E_{c}>\left(E_{\eta} E_{h}\right)^{1 / 2}$ полная стабильность будет иметь место при значениях $\alpha$, которые могут превысить 1 .

Согласно (28)-(30) граничное значение тока, после превышения которого лавинообразного перехода в нормальное состояние не происходит, равно

$$
\iota_{q, 0}=\left(E_{h} / E_{c}\right)^{1 /(n+1)} .
$$

Очевидно, что для всех $E_{h}>E_{c}$ будут иметь место стабильные закритические режимы, так как в этом случае $\iota_{q, 0}>1$.

Выписанные критерии показывают, что вероятность существования режима ввода тока без его срыва даже в закритической области будет тем выше, чем меньше $E_{\eta}$ или больше $E_{h}$. Подчеркнем, что в рамках существующей теории тепловой стабилизации описать подобные состояния не представляется возможным. Тем самым теорию тепловой стабилизации следует модернизировать с учетом крипа магнитного потока.

\section{Выводы}

Показано существование характерных значений напряженности электрического поля, которые при крипе магнитного потока влияют на степень однородности распределения электрического поля внутри технических сверхпроводников, крутизну нарастания их вольтамперных характеристик. Эти значения зависят от скорости ввода тока, геометрических размеров ленты, критических свойств сверхпроводника, условий передачи тепла, особенностей деления тока между сверхпроводником и стабилизирующим покрытием. Они лежат в основе механизмов формирования вольт-амперных характеристик технических сверхпроводников. Записаны критерии существования стационарных вольт-амперных характеристик с положительным наклоном, сохраняющимся при нагреве сверхпроводника вплоть до температуры, равной критической. При данных режимах спонтанного развития токовой неустойчивости не происходит, так как транспортный ток устойчиво перетекает из сверхпроводника в стабилизирующую матрицу по мере его увеличения. При этом могут наблюдаться стабильные закритические режимы ввода тока, когда последний будет превышать критический ток технического сверхпроводника. Записаны условия возникновения токовых неустойчивостей с учетом неравномерного распределения температуры по сечению технического сверхпроводника. Показано, что токи возникновения неустойчивых состояний не увеличиваются пропорционально увеличению критического тока сверхпроводника. 
В основе этой закономерности лежит эффект тепловой деградации. Установлено, что неоднородное распределение температуры по сечению сверхпроводника зависит не только от его термического сопротивления, но и от его критических свойств сверхпроводника, влияние которых будет возрастать с улучшением условий теплоотдачи. Введен безразмерный параметр $\gamma$, позволяющий оценить влияние критических свойств сверхпроводника на формирование его тепловых и электродинамических состояний. В результате при $\gamma \ll 1$ анализ теплоэлектродинамических состояний с хорошей степенью точности может быть проведен на основе нуль-мерного приближения.

Обсужденные особенности формирования теплоэлектродинамических состояний технических сверхпроводников необходимо учитывать при измерении их вольтамперных характеристик, а также при определении условий их устойчивой работоспособности. Полученные результаты особенно важны при анализе условий стабильности сверхпроводящего состояния интенсивно охлаждаемых токонесущих элементов, изготовленных на основе сверхпроводников с высокими значениями критической плотности тока.

\section{Список литературы}

[1] Уилсон М. Сверхпроводящие магниты. М.: Мир, 1985. $407 \mathrm{c}$.

[2] Гуревич А.Вл., Мини Р.Г., Рахманов А.Л. Физика композитных сверхпроводников. М.: Наука, 1987. 240 с.

[3] Romanovskii V.R., Watanabe K. // Superconductor: Science and Technology. 2006. Vol. 19. N 6. P. 541-550.

[4] Romanovskii V.R. // Cryogenics. 2002. Vol. 42. N 1. P. $29-37$.

[5] Polak M., Hlasnik I., Krempasky L. // Cryogenics. 1973. Vol. 13. N 12. P. 702.

[6] Архаров А.М., Лавров Н.А., Романовский В.Р. // ЖТФ. 2014. Т. 84. Вып. 8. С. 77-85.

[7] Romanovskii V.R., Watanabe K., Ken-ichiro Takahashi, Nishijima G., Awaji S. // Physica C - Superconductivity and its applications. 2004. Vol. 416. N 3-4. P. 126-136.

[8] Dresner L. // Cryogenics. 1993. Vol. 33. P. 900-909.

[9] Брехна Г. Сверхпроводящие магнитные системы. М.: Мир, 1976. $704 \mathrm{c}$.

[10] Bellis R.H., Iwasa Y. // Cryogenics. 1994. V. 34. N 2. P. 129-144.

[11] Seeber B. Handbook of Applied Superconductivity. Editor B. Seeber. Bristol: IOP Publishing, 1998. Vol. 1. P. 1067-1082. 